\title{
A SEQUENCE-TO-SEQUENCE TEMPORAL CONVOLUTIONAL NEURAL NETWORK FOR IONOSPHERE PREDICTION USING GNSS OBSERVATIONS
}

\author{
M. Kaselimi ${ }^{1}$, N. Doulamis ${ }^{1}$, A. Doulamis ${ }^{1}$, D. Delikaraoglou ${ }^{1}$ \\ ${ }^{1}$ School of Rural and Surveying Engineering, National Technical University of Athens, Athens 15780, Greece \\ mkaselimi@mail.ntua.gr; ndoulam@cs.ntua.gr; adoulam@cs.ntua.gr; ddeli@mail.ntua.gr
}

Commission III, WG III/2

KEY WORDS: Ionosphere variability, GNSS signal, convolutional neural networks, precise point positioning, total electron content

\begin{abstract}
:
This paper proposes a model suitable for predicting the ionosphere delay at different locations of receiver stations using a temporal $1 \mathrm{D}$ convolutional neural network $(\mathrm{CNN})$ model. CNN model can optimally addresses non-linearity and model complex data through the creation of powerful representations at hierarchical levels of abstraction. To be able to predict ionosphere values for each visible satellite at a given station, sequence-to-sequence (seq2seq) models are introduced. These models are commonly used for solving sequential problems. In seq2seq models, a sequential input is entered to the model and the output has also a sequential form. Adopting this structure help us to predict ionosphere values for all satellites in view at every epoch. As experimental data, we used global navigation satellite system (GNSS) observations from selected sites in central Europe, of the global international GNSS network (IGS). The data used are part of the multi GNSS experiment (MGEX) project, that provides observations from multiple navigation satellite systems. After processing with precise point positioning (PPP) technique as implemented with GAMP software, the slant total electron content data (STEC) were obtained. The proposed CNN uses as input the ionosphere pierce points (IPP) points coordinates per visible satellite. Then, based on outcomes of the ionosphere parameters, the temporal CNN is deployed to predict future TEC variations.
\end{abstract}

\section{INTRODUCTION}

Ionosphere variability is an intense and spatio-temporal varying phenomenon. Each global navigation satellite system (GNSS) signal is affected by the ionospheric variability in a different way, depending on signal's frequency (Hoque, Jakowski, 2007). As result, performance degradation in GNSS positioning is caused. Proper combination between the different signal frequencies or external ionospheric information, is useful to eliminate the ionospheric error and improve the performance in GNSS positioning. Applying external ionospheric information would be useful in a precise point positioning-real time kinematic (PPP RTK) processing scenario to enhance the integer ambiguity resolution (IAR) (Psychas et al., 2018) and improve both the performance and convergence time (Aggrey, Bisnath, 2019). In most cases, external ionosphere information is provided by the center for orbit determination in Europe (CODE) sparse ionosphere maps with spatial granularity of $\left(2.5^{\circ} \times 5.0^{\circ}\right)$, and 2-h temporal granularity, at global level (Schaer, 1997). Even if the corrections applied improve the accuracy achieved in positioning, an amount of noise caused by the ionosphere still remains. Hereby, we propose a Convolutional Neural Network (CNN) to create regional autoregressive total electron content (TEC) models. The proposed models referring to an area, could be applied to near the reference area stations or roving users, to correct the error caused by ionosphere variability.

$\mathrm{CNN}$ is a powerful tool for various computer vision and machine learning applications (Voulodimos et al., 2018). Unlike traditional shallow learning models, CNNs with their hidden layers and hyper-parameters, can successfully model complex data through the creation of powerful representations at hierarchical levels of abstraction. Nevertheless, a properly trained network is required with a large amount of data, in order to successfully learn suitable representation. CNNs have unique abilities for various applications such as image classification, segmentation and object detection for $2 \mathrm{D}$ images or $3 \mathrm{D}$ videos. On the other hand, recurrent neural networks (RNN) are popular for time series analysis and modelling (Kaselimi et al., 2020a). However, recent research has shown CNN's to be efficient at time series problems. Particularly, 1D CNN (Kiranyaz et al., 2019 ) is very effective in extracting important features. The 1D CNN configuration is applicable to the analysis of sensory (such as gyroscope or accelerometer) data time sequences, or to the analysis of any kind of signal data, such as audio and speech signals. 1D CNNs have recently been proposed and immediately achieved the state-of-the-art performance levels in various application domains such as detection early diagnosis in medical applications (Kiranyaz et al., 2019), anomaly detection, structural health monitoring and identification in power electronics and energy related applications (Kaselimi et al., 2019). They have simple and compact configuration, as they perform 1D convolutions, thus, they are excellent solution for real-time and low-cost applications.

\subsection{Background}

Among the empirical approaches, the broadcast NeQuick (Nava et al., 2008) and Klobuchar (Klobuchar, 1987) models are wellknown for estimating the ionospheric TEC parameters. GPS satellites broadcast the parameters of the Klobuchar ionospheric model for single frequency users. NeQuick is three-dimensional and time-dependent ionospheric model adopted by the European global navigation satellite system Galileo, in order to help its single-frequency users to compute the ionosphere parameter corrections. Additionally, the international reference ionosphere 
(IRI) model, provides monthly averages of various ionosphere parameters, at global scale (Jiang et al., 2019). The global ionosphere maps (GIM) are provided by the international GNSS service (IGS) in the IONospheric EXchange (IONEX) format. The ionosphere associate analysis centers (IAACs) compose the GIM products using TEC measurements derived from a worldwide network of hundreds of GNSS receiver stations. In addition, measurements using very long baseline interferometry (VBLI) and satellite altimetry techniques are also deployed among with other data, such as GNSS data, to obtain the ionosphere parameters (Alizadeh et al., 2013). The aforementioned models are global representations of TEC values, however, their accuracy imposes a crucial restriction arising, for instance, from the adoption of a simple Klobuchar model or the inadequacy of the NeQuick model to handle successfully extreme ionospheric conditions and predict the irregular TEC behavior. Most of these global maps fail to model accurately the high level of dependencies needed when down sampling is necessary to move from global to regional level.

There are various attempts focusing on mapping the ionosphere at local level, using tomographic-based methods (dos Santos Prol et al., 2018). In addition, refractive gradients in the ionosphere derived from the GNSS low earth orbit (LEO) satellites are used for accurate determination of atmospheric parameter profiles, using radio occultation (RO) technique (Norman et al., 2018). Multi-GNSS receivers can also contribute to accurate ionosphere modelling (Zhao et al., 2018). Using autoregressive models is a common approach towards enhancing the modelling of the ionospheric delays at a regional level (Kong et al., 2017). However, linear methods for the approximation of ionospheric delays are insufficient in describing the real ionosphere conditions, therefore, applying machine learning techniques for modelling the highly non-linear and complex ionosphere conditions is becoming imperative. Artificial neural networks (ANNs) techniques can successfully deal with short-term forecasting (Baliyan et al., 2015), (Doulamis et al., 2003). Due to the recent widespread advancements in machine learning, various studies introduce these methods to develop the suitable TEC prediction methods. Among these studies, techniques including shallow learning methods, such as support vector machine (SVM) (Zhang et al., 2019b), the nonlinear radial basic function (RBF) neural network (Huang, Yuan, 2014), (Yilmaz et al., 2009) and neural network (Ou et al., 2012), (Feng et al., 2019) have been introduced. RNNs can sufficient handle TEC observations and are able to be adapted in order to learn the temporal dependencies from context (Kaselimi et al., 2020b) and monitor the irregular ionospheric structure. The recurrent networks "memorize" previous term correlations in a timeseries, thus, they model sequences of data, as in ( $\mathrm{Su}$ et al., 2019), (Sun et al., 2017), (Tang et al., 2020), where parameters such as $10.7 \mathrm{~cm}$ solar flux and magnetic activity indices are anticipated, to achieve better modelling accuracy. Most of them targeted in analysis and prediction based on traditional autoregressive models (i.e., the autoregressive (AR) and the autoregressive moving average (ARMA) model), or recently in deep learning schemes, for instance the recurrent neural networks (RNN), which are straightforward applicable to the detection of various patterns in timeseries data. However, relevant studies applying $\mathrm{CNN}$ structures for timeseries ionosphere prediction are limited. To our knowledge, the studied methods in literature, focus on TEC prediction based on estimates derived from TEC models, such as GIM maps. In contrast to this approach, our method predicts ionosphere delays for each visible GNSS satellite individually.
Table 1. The stations' information (names, location (longitude and latitude) and country) for six selected sites of the IGS network.

\begin{tabular}{cccc}
\hline Site & Latitude $^{\circ}$ & Longitude $^{\circ}$ & Country \\
\hline bor1 & 52.27695 & 17.07345 & Poland \\
ganp & 49.03471 & 20.32293 & Slovakia \\
graz & 47.06713 & 15.49348 & Austria \\
leij & 51.35398 & 12.37410 & Germany \\
pots & 52.37929 & 13.06609 & Germany \\
wtzz & 49.14421 & 12.87890 & Germany \\
\hline
\end{tabular}

\subsection{Contribution}

The ionosphere varies with latitude, longitude, universal time, season, solar cycle and magnetic activity (Schunk, 1983). The advantage of our proposed model lies in ability of adapting all these variables as inputs to the network and in addition, easily expand the input features if necessary.

Furthermore, sequence-to-sequence (seq2seq) models (Sutskever et al., 2014) are introduced to successfully catch the sequential character of TEC values and thus predict them for each visible satellite at a given station. In seq 2 seq models, the input is a time sequence of data, and the output is also a time sequence. Adopting this structure help us to predict ionosphere values for all satellites in view at every epoch.

This work is further attempts:

- to define the characteristics of the GNSS ionospheric delays, especially at mid-latitudes, using multiple frequency observations,

- to derive potentially suitable models which can eventually be used to apply ionosphere corrections for single station and single frequency techniques,

- propose an efficient model for constructing dynamic changing, regional TEC models.

\section{GNSS AND IONOSPHERIC VARIABILITY}

The ionosphere is that part of the earth's upper atmosphere (extending roughly between 70 and $600 \mathrm{~km}$ ) with sufficient concentration of free electrons to affect the pro-pagation of electromagnetic waves. Its existence is primarily the result of the absorption of solar ultraviolet radiation in that part of the atmosphere which in turn reacts to produce free electrons and ions. Total Electron Content (TEC) is often used to describe ionospheric variability and is space and time varying. it is widely known that ionosphere exhibits significant variations with:

- latitude and longitude: the most disturbed region is the aurora zone (between $60^{\circ}$ to $70^{\circ} \mathrm{N}$ geomagnetic latitude) followed by the polar zone $\left(>70^{\circ} \mathrm{N}\right)$, while irregularities at equatorial ionosphere follow,

- local time: during the sunny hours of the day, the ionospheric condition variations are higher than those in nighttime period,

- solar cycle and geomagnetic activity. 
The GNSS signal travels through the ionosphere and due to the severe spatio-temporal changes of the electron density, significant disruptions on the traveling GNSS radio wave are caused. As a consequence of ionosphere's dispersive nature, is that for different carrier wave frequencies, different delays are caused. This fact provides one of the greatest advantages of a dualfrequency receiver over the single-frequency receivers: appropriate mathematical combinations among the different frequencies can eliminate ionosphere delay error. Hence, the use of multiple navigation signals of distinct center frequency transmitted from the same GNSS satellite allows direct observation and removal of the great majority of the ionospheric delay. It is worth mentioned that, the severity of the ionosphere's effect on a GNSS signal depends on the amount of time that signal spends traveling through it. A signal originating from a satellite near the observer's horizon (low satellite elevation) must pass through a larger amount of the ionosphere to reach the receiver than does a signal from a satellite near the observer's zenith (high satellite elevation). Thus, the longer the signal is in the ionosphere, the greater the ionosphere's effect on it.

\section{THE PRE-PROCESSING STARATEGY}

In this paper, STEC values are extracted using the undifferenced and uncombined observations in dual-frequency PPP processing. The observation equations are written as (Zhang et al., 2019a):

$$
\begin{array}{r}
P_{(f i)_{r}}^{s}=\rho_{r}^{s}+c \cdot(d t+d T)+D_{\text {trop }}+I_{(f i)}+ \\
c \cdot\left(d_{(f i)_{r}}-d_{f i}^{s}\right)+\xi_{P_{(f i)}}^{s} \\
\phi_{(f i)_{r}}^{s}=\rho_{r}^{s}+c \cdot(d t+d T)+D_{\text {trop }}-I_{(f i)}+ \\
\lambda N_{(f i)}^{s}+c \cdot\left(\delta_{(f i)_{r}}-\delta_{f i}^{s}\right)+\zeta_{\phi(f i)}^{s}
\end{array}
$$

where $P_{(f i)_{r}}^{s}$ and $\phi_{(f i)_{r}}^{s}$ are the pseudorange and carrier phase observables, respectively, between a receiver $r$ and a satellite $s$ in a frequency band $f_{i} ; \rho_{r}^{s}$ is the geometric distance between the satellite at the emission time and the receiver antenna at the reception time; c is the speed of light; $d t$ and $d T$ are the receiver and satellite clock offsets from the reference satellite system time, respectively; $d_{(f i)_{r}}$ is the frequency-dependent receiver uncalibrated code delay (UCD) while $d_{f i}^{s}$ is the frequency dependent satellite UCD (in seconds); $D_{\text {trop }}$ is troposphere delay (in meters); $I_{(f i)}$ is the slant ionospheric delay on the frequency $f i$ (in meters); $\delta_{(f i)_{r}}$ and $\delta_{f i}^{s}$ are the frequency-dependent receiver and satellite uncalibrated phase delay, respectively (in seconds); $N_{(f i)}^{s}$ denotes the ambiguity; $\xi_{P_{(f i)}}^{s}$ and $\zeta_{\phi_{(f i)}}^{s}$ are unmodelled errors including the sum of measurement noise and multi-path error for pseudorange and carrier phase observations respectively. For dual-frequency GPS receivers (G), and assuming the frequencies $f_{1}$ and $f_{2}$ noted as " 1 " and " 2 ", respectively, the Equation (1) is:

$$
\begin{aligned}
P_{1_{r}}^{G}=\rho_{r}^{G}+c \cdot(d t+ & \left.d T^{G}\right)+D_{\text {trop }}+I_{1}+ \\
& +c \cdot\left(d_{1_{r}}-d_{1}^{G}\right)+\xi_{P_{1}}^{G}
\end{aligned}
$$

$$
\begin{array}{r}
P_{2_{r}}^{G}=\rho_{r}^{G}+c \cdot\left(d t_{r}+d t^{G}\right)+T+I_{2}+ \\
+c \cdot\left(d_{2_{r}}-d_{2}^{G}\right)+\epsilon_{P_{2}}^{G}
\end{array}
$$

thus, the code biases are commonly referred as differential code biases (DCBs): $D C B=D C B_{P 1 / P 2}=d_{1}-d_{2}$ and also, given that $\gamma_{2}=f_{1}^{2} / f_{2}^{2}$, we have:

$$
\begin{array}{r}
d_{1}=d_{I F}+1 /\left(1-\gamma_{2}\right) \cdot D C B \text { and } \\
d_{2}=d_{I F}+\gamma_{2} /\left(1-\gamma_{2}\right) \cdot D C B
\end{array}
$$

and then,

$$
\begin{array}{r}
d_{1_{r}}-d_{1}^{G}=d_{(I F)_{r}}-d_{I F}^{G}+ \\
+\frac{1}{\left(1-\gamma_{2}\right)} \cdot\left(D C B_{r}-D C B^{s}\right)
\end{array}
$$

$$
\begin{array}{r}
d_{2_{r}}-d_{2}^{G}=d_{(I F)_{r}}-d_{I F}^{G}+ \\
+\frac{\gamma_{2}}{\left(1-\gamma_{2}\right)} \cdot\left(D C B_{r}-D C B^{s}\right)
\end{array}
$$

The term $I_{1}$ is grouped with differential code biases, thus:

$$
\tilde{I}_{1}=I_{1}-\frac{1}{\left(1-\gamma_{2}\right)} D C B^{s}+\frac{1}{\left(1-\gamma_{2}\right)} D C B_{r}
$$

In our case, the uncombined PPP (UPPP) model computes the ionosphere delay as unknown parameter, in contrast to the traditional ionosphere-free (IF) model which combines multiple frequency observations to eliminate the ionospheric error. However, for the estimation of TEC values using pseudorange and phase GNSS observables, the DCB biases should also considered, as $1 n s$ in a DCB, causes an $\sim 2.9 T E C U$ error in TEC estimation:

$$
\tilde{I}_{1}=M F_{I} \cdot v t e c-\frac{1}{\left(1-\gamma_{2}\right)} D C B^{s}+\frac{1}{\left(1-\gamma_{2}\right)} D C B_{r}
$$

Having the $D C B s$ corrected, the STEC can be converted into the vertical total electron content VTEC by applying a mapping function $M F_{I}$ (Bergeot et al., 2014):

$$
M F_{I}=\frac{\text { stec }}{v t e c}=\frac{1}{\left(1-\left(\frac{R_{e}}{R_{e}+h_{s}} \cos \theta\right)^{2}\right)^{1 / 2}}
$$

where $R_{e}$ is the mean Earth's radius; $\theta$ is the satellite's elevation angle; $h$ is the height of the ionospheric layer and usually has been taken about $350 \mathrm{~km}$.

\section{IONOSPHERE PREDICTION PROBLEM FORMULATION BASED ON GNSS OBSERVATIONS}

The combination of observations from different satellites (distinguished by their respective pseudorandom noise -PRN) and 


\section{D Tensor Input}

(rows, columns, depth) (features, timesteps, PRNs)
Temporal Convolutional Neural Network

Conv1D Conv1D

$(60,5) \quad(120,5)$
Flatten

\section{Dense}

(100)
(1)
Output

(rows, columns)

(timesteps, PRNs)
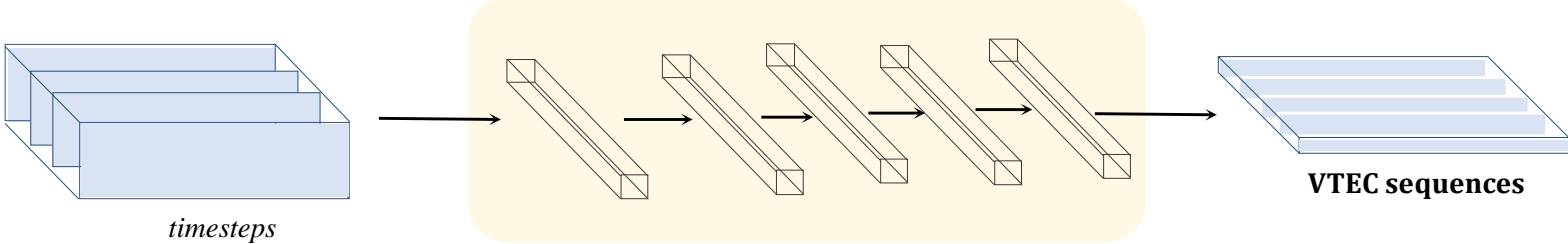

VTEC sequences

Figure 1. Temporal CNN network architecture.

different frequency bands, resulting in the successful estimation of VTEC values. According to the typical ionospheric prediction model, it is assumed that the ionosphere is a thin shell above the Earth, with the single-layer height ranging between 300 and $550 \mathrm{~km}$. The intersection between the line of sight of a GNSS satellite and this shell is called the Ionospheric Pierce Point (IPP). At different epochs, at every IPP point, each individual satellite provides a different VTEC measurement. Our model takes into consideration previous TEC values and predicts the future ones. Thus, the proposed CNN model is a nonlinear autoregressive model:

$$
v t e c_{t+1}=f\left(\phi_{I P P}\{1: t\}, \lambda_{I P P}\{1: t\}, \operatorname{vtec}\{1: t\}\right)
$$

\section{THE PROPOSED CNN FOR TEC MODELLING}

\subsection{Seq2seq temporal 1D CNN regression model for TEC prediction}

At first, we model the unknown function $f(\cdot)$ through a feedforward neural network. Assuming $L$ hidden neurons and a linear output layer, the estimate $v t e c^{s}$ every time, is given by (Doulamis et al., 2003):

$$
\begin{gathered}
v t e c^{s}(t)=\mathbf{u}^{T}(t) \cdot \mathbf{v} \\
\mathbf{u}(t)=\left[\begin{array}{c}
u_{1}(t) \\
\vdots \\
u_{L}(t)
\end{array}\right]=\left[\begin{array}{c}
\tanh \left(\mathbf{w}_{1}^{T} \cdot \mathbf{x}(t)\right) \\
\vdots \\
\tanh \left(\mathbf{w}_{L}^{T} \cdot \mathbf{x}(t)\right)
\end{array}\right]
\end{gathered}
$$

$\tanh (\cdot)$ is the hyperbolic tangent, $x(t)$ is the input vector (see Equation 11), $w_{i}, i=1, \ldots, L$, are the weights connect the input vector $\mathbf{x}(t)$, with the i-th hidden neuron. The term $u(t)$ gathers the outputs of all L hidden neurons $u_{i}$ with values ranging between -1 and 1. Similarly, v -vector (see Equation 12) encloses weights that connect the hidden neurons with the output neuron.

Convolutional Neural Network (CNN) is a more complex variant than a simple feed forward neural network, has its origins in the field of computer vision, but nowadays, due to its popularity, is spread in a wide range of applications. Its name is derived from the type of hidden layers that it consists of. Typically, the hidden layers of a CNN consist of convolutional layers, pooling layers, fully connected layers, and normalization layers. In case of one-dimensional convolutional neural networks, the major advantage is the model's low computational complexity since the only operation with a significant cost is a sequence of 1D convolutions which are weighted sums of two 1D arrays. For a given layer $l$, each (hidden or output) unit $z_{k}^{l}$ in such a network, computes a function given by:

$$
z_{k}^{l}=f\left(b_{k}^{l}+\sum_{j=1}^{l-1}\left(w_{k j}^{l-1}, s_{j}^{l-1}\right)\right)
$$

where $x_{k}^{l}$ is defined as the output/hidden unit, $b_{k}^{l}$ is defined as the bias of the $k^{t h}$ neuron at layer $l-1, s_{j}^{l-1}$ is the output of the $j^{\text {th }}$ neuron at layer $l-1, w_{k j}^{l-1}$ is the kernel from the $j^{\text {th }}$ neuron at layer $l-1$ to the $k^{\text {th }}$ neuron at layer $l$.

\subsection{The CNN network configuration for TEC modelling}

As illustrated in Figure 1, our proposed network configuration consists of two convolutional layers. The first convolutional layer has 60 filters of height 5 and the second one 120 filters of the same size. Then, a layer that flattens the output of convolutional neural network layer follows. Dense layer assigns a linear operation in which the input of the previous layer is connected to the output by a weight and is followed by a non-linear activation function. In our case, the activation function is the hyperbolic tangent function. For the training, the number of epochs is selected 600. The optimizer is the Adam and the loss function is the mean squared error.

\section{EXPERIMENTAL RESULTS}

\subsection{Dataset}

The experimental setup consists of a selected small group of permanent GNSS stations of the global network of International GNSS Service (IGS). The time granularity of the data is 30 s. Table 1 shows the position of the selected ground receiver IGS stations (Montenbruck et al., 2017), across central Europe in close proximity to each other.

The GAMP software (Zhou et al., 2018), a secondary development software based on RTKLIB (Kaselimi et al., 2018) has been used for precise point positioning. We have used the uncombined PPP (UPPP) ionosphere constraint model to estimate the slant TEC values as unknown parameters. The observation and navigation files, the precise orbit and clock information, the antenna phase centre corrections for both receivers network and satellites, as well as ocean tide loading coefficients and the differential code biases are processed using the GAMP software in static PPP mode. 
Table 2. A comparative table that summarizes the performance achieved by the CONV1D network in predicting the VTEC (TECU) values over the site stations (bor1, ganp, graz, leij, pots and wtzz).

\begin{tabular}{|c|c|c|c|c|c|c|c|c|c|c|c|c|}
\hline \multirow{2}{*}{$\begin{array}{c}\text { Site } \\
\text { Metric }\end{array}$} & \multirow[b]{2}{*}{$\overline{m a e}_{r}$} & \multicolumn{2}{|l|}{ bor1 } & \multirow[b]{2}{*}{$\overline{r m s e}_{r}$} & \multirow[b]{2}{*}{$\overline{m a e}_{r}$} & \multicolumn{2}{|l|}{ ganp } & \multirow[b]{2}{*}{$\overline{r m s e}_{r}$} & \multirow[b]{2}{*}{$\overline{m a e}_{r}$} & \multicolumn{2}{|l|}{ graz } & \multirow[b]{2}{*}{$\overline{r m s e}_{r}$} \\
\hline & & $\overline{\min }_{r}$ & $\overline{\max }_{r}$ & & & $\overline{\min }_{r}$ & $\overline{\max }_{r}$ & & & $\overline{\min }_{r}$ & $\overline{\max }_{r}$ & \\
\hline CONV1D & 0.91 & 0.02 & 2.41 & 1.09 & 0.93 & 0.06 & 2.21 & 1.08 & 0.88 & 0.01 & 2.25 & 1.03 \\
\hline LSTM & 0.98 & 0.04 & 3.05 & 1.18 & 1.24 & 0.02 & 2.88 & 1.45 & 1.09 & 0.03 & 2.76 & 1.26 \\
\hline ARMA & 1.41 & 0.01 & 3.76 & 1.76 & 1.63 & 0.02 & 3.94 & 2.03 & 1.70 & 0.04 & 3.85 & 2.03 \\
\hline AR & 1.91 & 0.04 & 4.40 & 2.29 & 1.85 & 0.02 & 4.28 & 2.25 & 1.77 & 0.02 & 3.98 & 2.13 \\
\hline Site & & leij & & & & pots & & & & wtzz & & \\
\hline Metric & $\overline{m a e}_{r}$ & $\overline{\min }_{r}$ & $\overline{\max }_{r}$ & $\overline{r m s e}_{r}$ & $\overline{m a e}_{r}$ & $\min _{r}$ & $\overline{\max }_{r}$ & $\overline{r m s e}_{r}$ & $\overline{m a e}_{r}$ & $\overline{\min }_{r}$ & $\overline{\max }_{r}$ & $\overline{r m s e}_{r}$ \\
\hline CONV1D & 0.71 & 0.02 & 2.07 & 0.88 & 0.84 & 0.02 & 2.39 & 1.03 & 0.83 & 0.03 & 2.25 & 0.99 \\
\hline LSTM & 0.93 & 0.02 & 2.66 & 1.14 & 1.19 & 0.01 & 3.52 & 1.42 & 1.03 & 0.04 & 2.68 & 1.23 \\
\hline ARMA & 1.63 & 0.01 & 3.96 & 1.98 & 1.71 & 0.01 & 4.12 & 2.07 & 1.54 & 0.01 & 3.78 & 1.91 \\
\hline AR & 1.87 & 0.01 & 4.22 & 2.22 & 1.94 & 0.01 & 4.33 & 2.30 & 1.79 & 0.01 & 4.10 & 2.15 \\
\hline
\end{tabular}

\subsection{Performance Evaluation}

Table 2 provides comparative results for the proposed CONV1D method and (i) the recurrent LSTM (Long Short-term Memory) network (Kaselimi et al., 2020b), (ii) the AR model (Autoregressive) and (iii) the ARMA model (Autoregressive moving Average) model. AR and ARMA models are traditional stochastic models used for timeseries modeling, while recurrent neural networks allow previous outputs to be used as inputs for the next step, enhancing their recurrent character and their ability to successfully deal with sequential data. For each individual PRN, we compute the absolute difference between the ground truth $\widehat{v t e c}_{r, t}$ value, as computed using the GAMP software with PPP processing, and the respective VTEC values as being estimated from our Conv1d model $v t e c_{r, t}^{s_{i}}$. Thus, every epoch $t$, the absolute difference is $\left|v t e c_{r, t}^{s_{i}}-\widehat{v t e c}_{r, t}^{s_{i}}\right|$. The metrics for comparison where selected to be:

(i) the mean absolute error $\overline{m a e}_{r}$, which is the average value of the mean absolute errors $M A E_{r}^{s_{i}}$ per individual PRN $s_{i}$,

$$
\begin{aligned}
\overline{\mathrm{mae}}_{r} & =\frac{1}{S} \sum_{s_{i}=1}^{S} M A E_{r}^{s_{i}} \\
& =\frac{1}{S} \sum_{s_{i}=1}^{S}\left(\frac{\sum_{t=1}^{T}\left|v t e c_{r, t}^{s_{i}}-\widehat{v t e c}_{r, t}^{s_{i}}\right|}{T}\right)
\end{aligned}
$$

(ii) $\overline{\min }_{r}$ is the average of all $s_{i}$ minimum difference values per station,

$$
\overline{\min }_{r}=\frac{1}{S} \sum_{s_{i}=1}^{S}\left(\min _{\forall t \in T}\left(\left|v t e c_{r, t}^{s_{i}}-\widehat{\operatorname{vtec}}_{r, t}^{s_{i}}\right|\right)\right)
$$

(iii) in accordance to $\overline{\min }_{r}$ metric, $\overline{\max }_{r}$ is the average of all $s_{i}$ maximum differences values per station, and

$$
\overline{\max }_{r}=\frac{1}{S} \sum_{s_{i}=1}^{S}\left(\max _{\forall t \in T}\left(\left|v t e c_{r, t}^{s_{i}}-\widehat{\operatorname{vtec}}_{r, t}^{s_{i}}\right|\right)\right)
$$

(iv) $\overline{r m s e}_{r}$ is the average of $s_{i}$ root mean squared error $R M S E_{r}^{s_{i}}$ of all PRNs (satellites) per station.

$$
\begin{aligned}
\overline{r m s e}_{r} & =\frac{1}{S} \sum_{s_{i}=1}^{S} R M S E_{r}^{s_{i}} \\
& =\frac{1}{S} \sum_{s_{i}=1}^{S}\left(\sqrt{\frac{1}{T-1} \sum_{t=1}^{T}\left(\operatorname{vtec}_{r, t}^{s_{i}}-\widehat{\operatorname{vtec}}_{r, t}^{s_{i}}\right)^{2}}\right)
\end{aligned}
$$

The $\overline{m a e}_{r}$ error ranges between 0.71 and 0.91 for our proposed CONV1D method, which is better than $1 T E C U$. As regards the recurrent LSTM method, the $\overline{m a e}_{r}$ error is 0.93 to $1.24 T E C U$, while for the autoregressive methods, the respective mean absolute error is greater than 1.5 TECU. The $\overline{r m s e}_{r}$ values for CONV1D are between 0.88 and 1.09 , while in the other methods used for comparison, the results shown values greater than $1 T E C U$. As derived from the results, CONV1D extracts the temporal information and models successfully the TEC sequential problem.

Figure 2 shows the estimated TEC timeseries for each individual PRN for bor1 station for a single day of observations. Satellites with PRNs G01, G08, G11, G17 and G27 have the best performance, while G16 and G23, appear the worst one. Overall, as noticed, the predicted values are close to the estimated ones and the CONV1D network can successfully model TEC values per PRN.

Figure 3 shows the MAE results for two different test sets. Here, we can notice that the proposed results with the CONV1D method achieve slightly better performance than the traditional recurrent networks used for timeseries modelling.

Figure 4 illustrates the mean VTEC values at every site, as obtained from NeQuick, IRI2001 and GIM TEC estimates compared to the ground truth values (GRD) and CONV1D TEC predictions, during the day. The maximum VTEC values appeared the time duration between 8:00 and 12:00 A.M. In most cases, the CONV1D values are similar to those of GPS TEC derived values. This is as it expected, because the CONV1D model has been trained using as training set these values. However, it is noted that our model underestimates VTEC values, showing lower values than those of GPS TEC. GIM-aided TEC values are also close to CONV1D derived TEC values, which is also expected as the processing PPP strategy uses GIM TEC values for input (ionosphere constrained model). Finally, NeQuick and IRI2001 values show wider variability during the day with higher maximum and lower minimum values, compared those of GIM and GPS TEC methods. 

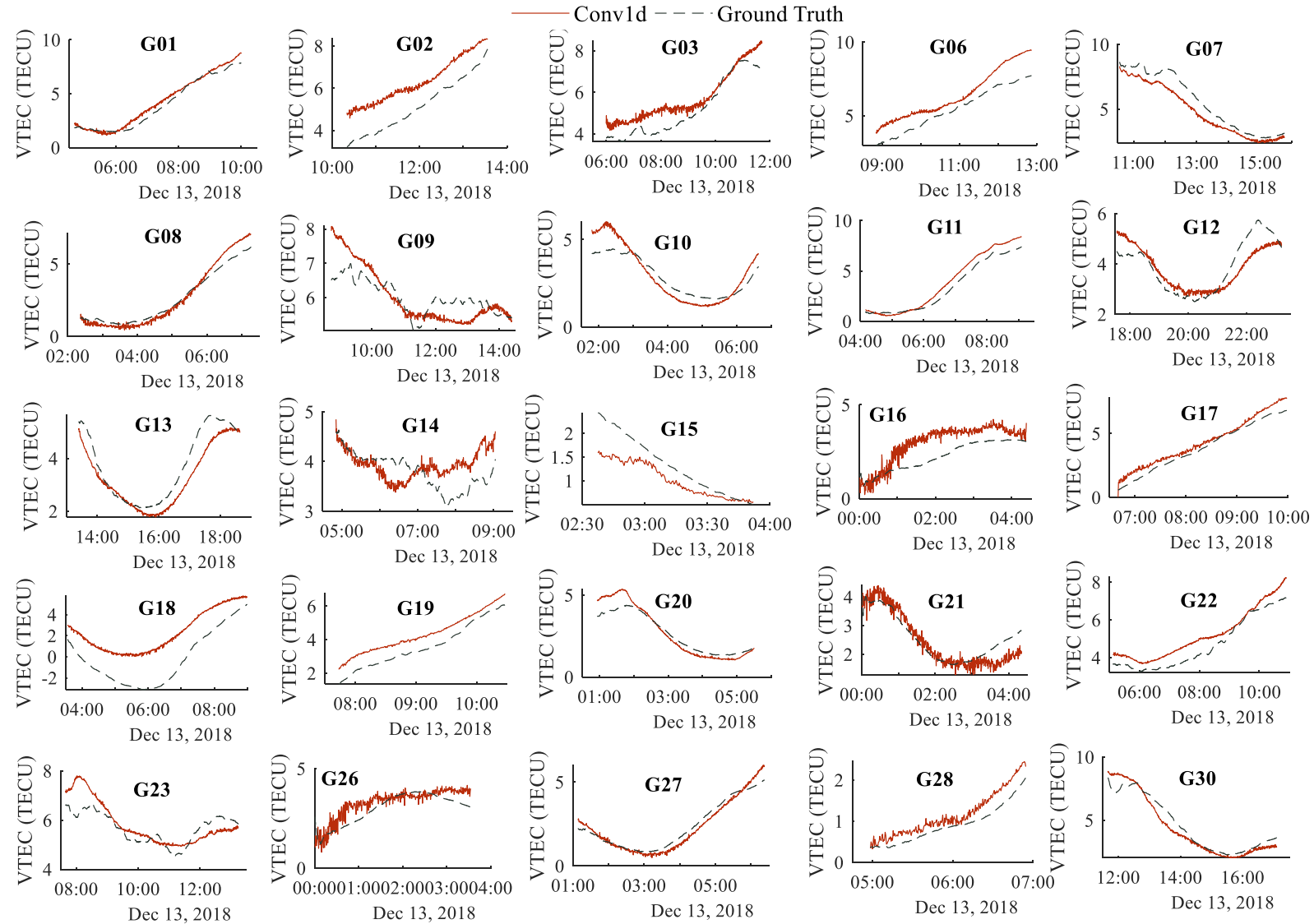

Figure 2. Conv1d versus ground truth per satellite PRN for bor1 station.

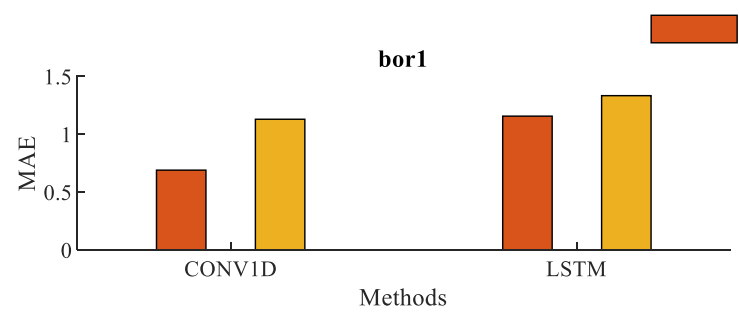

Test set 1
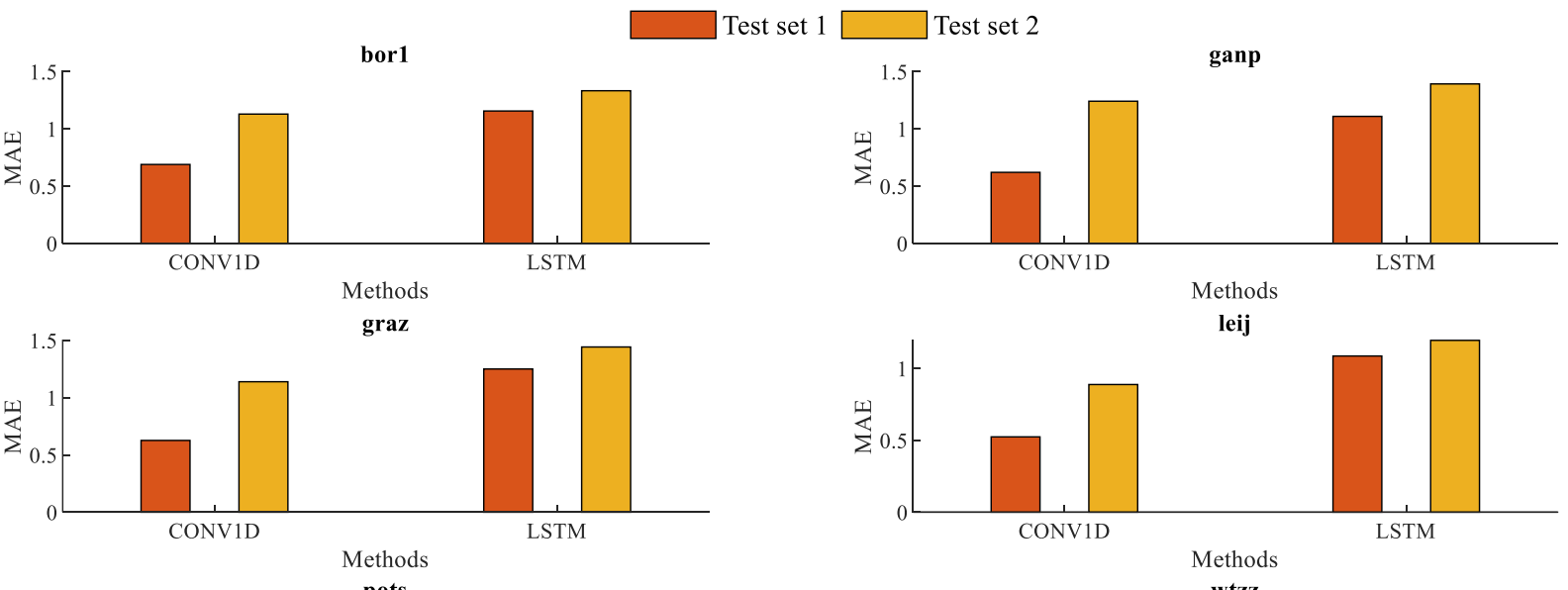

leij
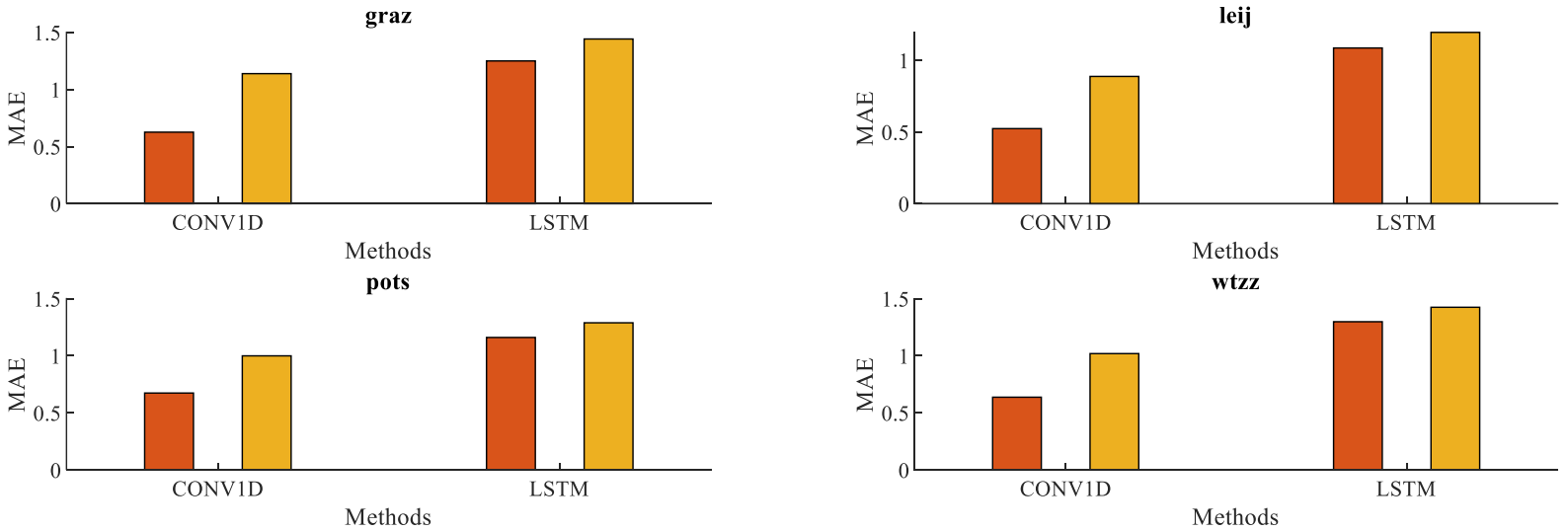

Figure 3. Conv1d versus ground truth per satellite PRN. 

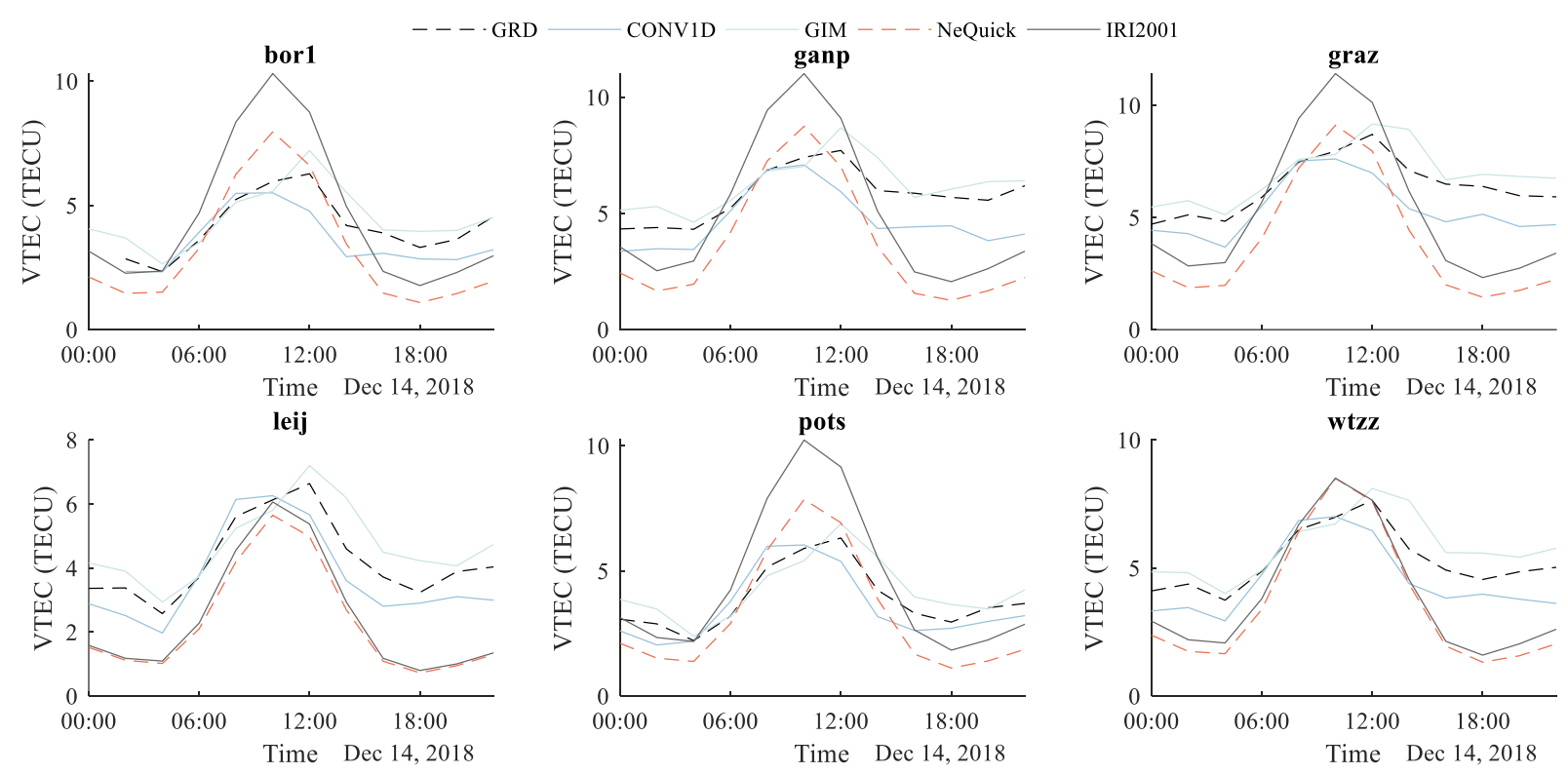

Figure 4. Conv1d versus ground truth per satellite PRN.

\section{CONCLUSION}

In this paper, we propose a non-linear autoregressive network based on convolutions for TEC modelling. Temporal convolutional neural networks can successfully model sequential data. In contrast to the recurrent neural networks that traditionally have been used for timeseries modelling, our CONV1D method except for higher accuracy, present lower complexity since the only operation with a significant cost is a sequence of $1 \mathrm{D}$ convolutions which are weighted sums of two 1D arrays. The aim of the paper, is to define the characteristics of the GNSS ionospheric delays, especially at mid-latitudes, using multiple frequency observations and data from multiple PRNs. Also, the paper aiming at derive models that apply accurate ionosphere TEC corrections, to increase the accuracy and convergence time for single station and single frequency techniques. propose an efficient model for constructing dynamic changing, regional TEC models. The experimental results indicate that mean absolute error ranges between 0.71 and 0.91 for the CONV1D method, which means that the error is better than $1 T E C U$.

\section{ACKNOWLEDGEMENTS}

Financial support has been provided by the Innovation and Networks Executive Agency (INEA) under the powers delegated by the European Commission through the Horizon 2020 program "PANOPTIS-Development of a decision support system for increasing the resilience of transportation infrastructure based on combined use of terrestrial and airborne sensors and advanced modelling tools," Grant Agreement number 769129.

\section{REFERENCES}

Aggrey, J., Bisnath, S., 2019. Improving GNSS PPP convergence: The case of atmospheric-constrained, multi-GNSS PPPAR. Sensors, 19(3), 587.

Alizadeh, M. M., Wijaya, D. D., Hobiger, T., Weber, R., Schuh, H., 2013. Ionospheric effects on microwave signals. Atmospheric effects in space geodesy, Springer, 35-71.
Baliyan, A., Gaurav, K., Mishra, S. K., 2015. A review of short term load forecasting using artificial neural network models. Procedia Computer Science, 48, 121-125.

Bergeot, N., Chevalier, J.-M., Bruyninx, C., Pottiaux, E., Aerts, W., Baire, Q., Legrand, J., Defraigne, P., Huang, W., 2014. Near real-time ionospheric monitoring over Europe at the Royal Observatory of Belgium using GNSS data. Journal of Space Weather and Space Climate, 4, A31.

dos Santos Prol, F., de Oliveira Camargo, P., HernándezPajares, M., Muella, M. T. d. A. H., 2018. A new method for ionospheric tomography and its assessment by ionosonde electron density, GPS TEC, and single-frequency PPP. IEEE Transactions on Geoscience and Remote Sensing, 57(5), 2571-2582.

Doulamis, A. D., Doulamis, N. D., Kollias, S. D., 2003. An adaptable neural-network model for recursive nonlinear traffic prediction and modeling of MPEG video sources. IEEE Transactions on Neural Networks, 14(1), 150-166.

Feng, J., Han, B., Zhao, Z., Wang, Z., 2019. A New Global Total Electron Content Empirical Model. Remote Sensing, 11(6), 706.

Hoque, M. M., Jakowski, N., 2007. Higher order ionospheric effects in precise GNSS positioning. Journal of Geodesy, 81(4), 259-268.

Huang, Z., Yuan, H., 2014. Ionospheric single-station TEC short-term forecast using RBF neural network. Radio Science, 49(4), 283-292.

Jiang, H., Liu, J., Wang, Z., An, J., Ou, J., Liu, S., Wang, N., 2019. Assessment of spatial and temporal TEC variations derived from ionospheric models over the polar regions. Journal of Geodesy, 93(4), 455-471.

Kaselimi, M., Doulamis, N. D., Delikaraoglou, D., Protopapadakis, E., 2018. Gnssget and gnssplot platforms - matlab guis for retrieving gnss products and visualizing gnss solutions. VISIGRAPP. 
Kaselimi, M., Doulamis, N., Voulodimos, A., Protopapadakis, E., Doulamis, A., 2020a. Context Aware Energy Disaggregation using Adaptive Bidirectional LSTM Models. IEEE Transactions on Smart Grid.

Kaselimi, M., Protopapadakis, E., Voulodimos, A., Doulamis, N., Doulamis, A., 2019. Multi-Channel Recurrent Convolutional Neural Networks for Energy Disaggregation. IEEE Access, 7, 81047-81056.

Kaselimi, M., Voulodimos, A., Doulamis, N., Doulamis, A., Delikaraoglou, D., 2020b. A Causal Long Short-Term Memory Sequence to Sequence Model for TEC Prediction Using GNSS Observations. Remote Sensing, 12(9), 1354.

Kiranyaz, S., Avci, O., Abdeljaber, O., Ince, T., Gabbouj, M., Inman, D. J., 2019. 1D convolutional neural networks and applications: A survey. arXiv preprint arXiv:1905.03554.

Klobuchar, J. A., 1987. Ionospheric time-delay algorithm for single-frequency GPS users. IEEE Transactions on aerospace and electronic systems, 325-331.

Kong, Y., Chai, H., Li, J., Pan, Z., Chong, Y., 2017. A modified forecast method of ionosphere vtec series based on arma model. 2017 Forum on Cooperative Positioning and Service (CPGPS), IEEE, 90-95.

Montenbruck, O., Steigenberger, P., Prange, L., Deng, Z., Zhao, Q., Perosanz, F., Romero, I., Noll, C., Stürze, A., Weber, G., Schmid, R., MacLeod, K., Schaer, S., 2017. The Multi-GNSS Experiment (MGEX) of the International GNSS Service (IGS) - Achievements, prospects and challenges. Advances in Space Research, 59(7), 1671 - 1697.

Nava, B., Coisson, P., Radicella, S., 2008. A new version of the NeQuick ionosphere electron density model. Journal of Atmospheric and Solar-Terrestrial Physics, 70(15), 1856-1862.

Norman, R., Carter, B. A., Healy, S., Culverwell, I., Von Engeln, A., Le Marshall, J., Younger, J., Cate, A., Zhang, K., 2018. Ionospheric Regions Producing Anomalous GNSS Radio Occultation Results. IEEE Transactions on Geoscience and Remote Sensing, 56(12), 7350-7358.

Ou, M., Zhang, H.-B., Liu, D., Yu, X., Zhen, W.-M., 2012. Gim-ingested nequick model applied for gps single frequency ionospheric correction in china. ISAPE2012, IEEE, 652-655.

Psychas, D., Verhagen, S., Liu, X., Memarzadeh, Y., Visser, H., 2018. Assessment of ionospheric corrections for PPP-RTK using regional ionosphere modelling. Measurement Science and Technology, 30(1), 014001.

Schaer, S., 1997. How to use CODE's global ionosphere maps. Astronomical Institute, University of Berne, 1-9.

Schunk, R., 1983. The terrestrial ionosphere. Solar-Terrestrial Physics, Springer, 609-676.

Su, K., Jin, S., Hoque, M., 2019. Evaluation of ionospheric delay effects on multi-GNSS positioning performance. Remote Sensing, 11(2), 171.

Sun, W., Xu, L., Huang, X., Zhang, W., Yuan, T., Yan, Y., 2017. Bidirectional lstm for ionospheric vertical total electron content (tec) forecasting. 2017 IEEE Visual Communications and Image Processing (VCIP), IEEE, 1-4.
Sutskever, I., Vinyals, O., Le, Q. V., 2014. Sequence to sequence learning with neural networks. Advances in neural information processing systems, 3104-3112.

Tang, R., Zeng, F., Chen, Z., Wang, J.-S., Huang, C.-M., Wu, Z., 2020. The Comparison of Predicting Storm-time Ionospheric TEC by Three Methods: ARIMA, LSTM, and Seq2Seq. Atmosphere, 11(4), 316.

Voulodimos, A., Doulamis, N., Doulamis, A., Protopapadakis, E., 2018. Deep learning for computer vision: A brief review. Computational intelligence and neuroscience, 2018.

Yilmaz, A., Akdogan, K., Gurun, M., 2009. Regional TEC mapping using neural networks. Radio Science, 44(3), 1-16.

Zhang, B., Chen, Y., Yuan, Y., 2019a. PPP-RTK based on undifferenced and uncombined observations: theoretical and practical aspects. Journal of Geodesy, 93(7), 1011-1024.

Zhang, Z., Pan, S., Gao, C., Zhao, T., Gao, W., 2019b. Support Vector Machine for Regional Ionospheric Delay Modeling. Sensors, 19(13), 2947.

Zhao, C., Yuan, Y., Zhang, B., Li, M., 2018. Ionosphere sensing with a low-cost, single-frequency, multi-GNSS receiver. IEEE Transactions on Geoscience and Remote Sensing, 57(2), 881892.

Zhou, F., Dong, D., Li, W., Jiang, X., Wickert, J., Schuh, H., 2018. GAMP: An open-source software of multi-GNSS precise point positioning using undifferenced and uncombined observations. Gps Solutions, 22(2), 33.

Revised January 2020 